\title{
A fast lightweight based deep learning for detecting macula fovea using ultra-widefield Fundus images
}

\author{
Han Wang ${ }^{1,2,3}$, Jie Yang ${ }^{4,5}$, Yaoyang Wu ${ }^{5}$, Wencai Du ${ }^{6}$, Simon Fong ${ }^{5}$, Feng \\ Wu $^{3}$, Yutong Duan ${ }^{3}$, Xiaoping Yao ${ }^{7}$, Xiaoshu Zhou ${ }^{2}$, Qingqian Li ${ }^{8}$, Chen \\ Lin $^{9}$, Jiang Liu ${ }^{10}$, and Lina Huang ${ }^{11}$ \\ ${ }^{1}$ Institute of Data Science, City University of Macau, Macau 999078 \\ ${ }^{2}$ School of Computer, Beijing Institute of Technology Zhuhai, China 519000 \\ ${ }^{3}$ Zhuhai Institute of Advanced Technology Chinese Academy of Sciences, China 519000 \\ ${ }^{4}$ College of Artificial Intelligence, Chongqing IndustryTrade Polytechnic, China 408000 \\ ${ }^{5}$ Faculty of Science and Technology, University of Macau, Macau 999078 \\ ${ }^{6}$ University of Saint Joseph, Macau 999078 \\ ${ }^{7}$ Union hospital of Fujian Medical University, Fujian, China 353000 \\ ${ }^{8}$ Department of Ophthalmology, People's Hospital of Yantian District, Shenzhen, China \\ 518000 \\ ${ }^{9}$ Department of Ophthalmology, Shenzhen People's Hospital, Shenzhen, China 518000 \\ ${ }^{10}$ Department of Computer Science and Engineering, Southern University of Science \\ and Technology, Shenzhen, China 518000 \\ ${ }^{11}$ Jinan University Affiliated Shenzhen Aier Eye Hospital, China 518000
}

Email address: lina ${ }_{h} @ 126 . c o m$

Corresponding author:

Han Wang ${ }^{1,2,3}$

Jie Yang 4,5

Yaoyang $\mathrm{Wu}^{5}$

Wencai $\mathrm{Du}^{6}$

Email address: Geogedu@usj.edu.mo

Chen $\operatorname{Lin}^{9}$

Lina Huang ${ }^{11}$

Email address: D20092100037@cityu.mo

Email address: jie.yang@connect.um.edu.mo

Email address: yc07481@connect.um.edu.mo

Email address: linchen66@hotmail.com 


\begin{abstract}
Macula fovea detection is a crucial prerequisite towards screening and diagnosing macular diseases. Without early detection and proper treatment, any abnormality involving the macula may lead to blindness. However, with the ophthalmologist shortage and time-consuming artificial evaluation, neither accuracy nor effectiveness of the diagnose process could be guaranteed. In this project, we proposed a deep learning approach on ultra-widefield fundus (UWF) images for macula fovea detection. This study collected 2300 ultra-widefield fundus images from Shenzhen Aier Eye Hospital in China. Methods based on U-shape network (Unet) and Fully Convolutional Networks (FCN) are implemented on 1800 (before amplifying process) training fundus images, 400 (before amplifying process) validation images and 100 test images. Three professional ophthalmologists were invited to mark the fovea. A method from the anatomy perspective is investigated. This approach is derivedfrom the spatial relationship between macula fovea and optic disc center in UWF. A set of parameters of this method is set based on the experience of ophthalmologists and verified to be effective. Results are measured by calculating the Euclidean distance between proposed approaches and the accurate grounded standard, which is detected by Ultra-widefield swept-source optical coherence tomograph (UWF-OCT) approach. Through a comparation of proposed methods, we conclude that, deep learning approach of Unet outperformed other methods on macula fovea detection tasks, by which outcomes obtained are comparable to grounded standard method.
\end{abstract}

\title{
INTRODUCTION
}

The macula is located approximately at the retina center, which is in charge of the central vision of human. Macula fovea is concepted as the center of macula, which is composed by compact core cells. Fovea allows us to maintain a sharp, clear central vision and discriminate colorsDeka et al. (2015). Any kind of retinal disease abnormality deposit at the edge of macula, such as Age-related Macular Degeneration(AMD), Diabetic Macular Edema(DME), Diabetic Retinopathy(DR),etc. can be responsible for center vision blockage, maculopathy, and retinal detachmentDeka et al. (2015); Medhi and Dandapat (2016). Macular lesion is one of the most serious scenarios among maculopathies, which is an abrupt and irreversible modulation in vision and is the major cause of blindnessNussenblatt (2010); Bird (2020). Maculopathy could result in choroidal inflammation and retinal pigment epithelial detachment, with $63 \%$ of the patents develop hemorrhagic lesionsWalma and Schlaegel (1964), 7\% of the macula patents suffer central serous choroidopathy symptomsWalma and Schlaegel (1964), some shows vitreous hemorrhageKranias (1985), a disciform scar could occur in months or yearsBird (2020), and during said period about $60 \%$ of the time the patients' vision is impairedKleiner et al. (1988). New lesions always occur around the old onesGass and Wilkinson (1972). For patients who have been diagnosed of histo scars before, there is an approximately $1 / 4$ of the chances that they would have a macula recrudescence within 3 years of their first recoverySchlaegel Jr (1977). Detecting Macula fovea plays a vital role in retinal screening, early detection and timely treatments Deka et al. (2015), which would significantly help prevent this irreversible damageMedhi and Dandapat (2016). However, without the presence of pigmentation and hemorrhage, it is laborious to detect macula lesion membranesRivers et al. (1992). The deficient quantity of ophthalmologists is highly incompatible with the large number of patientsMedhi and Dandapat (2016).

Conventional methods are artificial diagnoses based on Fluorescein Angiography (FA), color fundus photographs, Optical Coherence Tomography (OCT) and Retinal Thickness Analyzer (RTA), where retinal imaging and bio-microscopy technology are includedMedhi and Dandapat (2016); Sinthanayothin et al. (2002). Such detecting process is time-consuming, cumbersome, costly, and always make patients physically uncomfortable. To meet the shortages of traditional approaches, the novel automated diagnose and computer-aided assessment methods are enhanced for fovea detection. Sinthanayothin et al. (2002) detected the fovea at the darkest area of the Optic disc (OD), they applied template matching skills in the view of increased pigmentation features around the foveaSinthanayothin et al. (2002). Singh etc. (2008) described a method derivedfrom distinct structures of appearance for fovea localization Singh et al. (2008). Sekhar etc. (2008) employed Hough transform and morphological operations to explore the spatial distribution of optic disk(OD) and macula fovea, based on which, their study introduced a novel approach for OD and fovea detectionSekhar et al. (2008). These mathematical computer-aided methods performed well but they are still time-consuming and labor-intensive. Thus, since advanced artificial intelligence technologies present great advantages in many different fields, researchers consider and implement it into the ophthalmology field. Sedai etc. (2017) presented an end-to-end pixelwise segmentation framework based on fully convolutional neural network(FCN) deep learning (DL) algorithms, they tested the model 
on 400 retinal fund images with an average error of $14 \pm 7$ pixelsSedai et al. (2017). Al-Bander etc. (2018) demonstrated a deep learning method based on convolutional neural network (CNN) algorithm to detect the fovea and OD. They also examined the results with accurate grounded standard marked by experienced ophthalmologists, in their study, high accuracies of $97 \%$ and $96.6 \%$ were presented for fovea and OD detection respectivelyAl-Bander et al. (2018). Bian etc. (2020) Proposed an automatic fovea localization, OD segmentation and glaucoma classification frame work based on U-shape network (Unet) architecture, their research acquired a 47.8 average distance for fovea detectionBian et al. (2020). DL methods perform a great performances in resource-saving and efficient-enhancement, which present a preeminent potential for macular diagnose clinically.

Ultra-widefield fundus (UWF) imaging is an essential improvement and supplement to multiple imaging paradigm of OCT and scanning laser ophthalmoscopy (SLO). UWF images apply $200^{\circ}$ panoramic photographing technique to acquire various retinal modalities, which consists of fundus autofluorescence(FAF), pseudocolor, indocyanine green angiography(ICGA), and fluorescein angiography (FA)Nagiel et al. (2016). With a larger view and richer information, Optos UWF provides novel insights for retinal diagnosesNagiel et al. (2016); Yang et al. (2019). Applications of deep learning technology in macula fovea detection on normal fundus images of $45^{\circ}$ field of view (FOV) have been validated by renowned researchersSedai et al. (2017); Al-Bander et al. (2018); Niemeijer et al. (2009), but there has still been few applications on UWF images. Yang(2019) proposed a joint method for OD and fovea localization based on spatially constrained Faster R-CNN in the context of UWF, which was an idea not been considered beforeYang et al. (2019). However, with the lack of open source UWF datasetsYang et al. (2021), most of the deep learning-based methods are pertained by $45^{\circ}$ fundus images.

This study proposes a DL method for fovea detection on 2300 UWF images based on a lightweight Unet architecture, where 100 UWF photographs are randomly chosen as the test dataset. In this research, three experienced ophthalmologists are invited to locate the fovea center manually. Comparison methods include but not exclusive to FCN (fully convolutional Network), and also an anatomy approach based on the spatial relationship between OD and fovea. Regarded results based on OCT-induced approach as the grounded principle, this research examines the efficiency, accuracy and stability of the proposed methods. Thus, contributions are concluded as the following. (1) A three-layer lightweight Unet architecture is proposed. (2) A set of parameters of anatomy-based method is experimented and verified to be effective. (3) Five approaches are experimented and compared using the collected dataset: FCN-based method, Unet-based method, anatomy-based method, OCT-induced method and manual approach executed by human experts, where a time indicator and ten statistical indexes are used as evaluation criteria. (4) Features of automated and non-automated methods are investigated. (5) The proposed Unet-based method is verified with performances of cost consumption, accuracy and stability, which exhibits a potential for wide application on macular fovea detection clinically in the future.

The structure of this paper is organized as following. Methodology and experimental setup are introduced in the section 2 . In section 3 we analyze the experimental results and extend discussions. The conclusion is presented in section 4.

\section{MATERIALS AND METHODS}

Figure 1 illustrates the workflow of our experiment. The process includes 6 steps: 1. Macula fovea labeling of the training and validation datasets; 2. Image resizing and amplifying; 3. FCN and Unet based deep learning model building; 4 . Model training; 5 . Macular fovea detection with all chosen methods; 6 . Measuring the detection error with Euclidean distance calculation rules.

\section{Datasets}

UWF imaging is recognized as a significant innovation in ophthalmology scanning and diagnosing. With a panoramic FOV of $200^{\circ}$ or above, the vasculature and peripheral retina can be observed. Thus, images are expected to be the standard-of-care for clinical diagnosesNagiel et al. (2016). Some of the normal fundus images are openly accessed online, like the STARE datasetGoldbaum et al. (1990), where 400 images are included and categorized by 14 retinal disease. Up until the point when this paper is written there is no open source of UWF images available, probably because UWF images are difficult to obtain, especially in the underdeveloped areasYang et al. (2021). The recognized UWF datasets are included as the DeepDR and other in-house data sets. DeepDR is from the IEEE International Symposium on Biomedical Imaging conference, which includes 256 UWF images and 2000 regular fundus images collected from Chinese 
patients. The in-house datasets are collected from hospitals for lesion detection research purposes, such as Diabetic Retinopathy (DR)Carmona et al. (2008), Rhegmatogenous Retinal Detachment (RRD) Carmona et al. (2008), Retinitis Pigmentosa (RP) Carmona et al. (2008), Idiopathic Macular Hole (MH)Fogel and Kvedar (2018) and Proliferative Diabetic Retinopathy (PDR)Nagasawa et al. (2019) detections. UWF datasets for MH, RP, RRD and PDR are collected from Tsukazaki Hospital, which includes images from normal people and patients with lesion Carmona et al. (2008); Fogel and Kvedar (2018); Nagasawa et al. (2019).

As the Table 1 shows, this research collected 2300 Optos images from Shenzhen Aier Eye Hospital (Guangdong, China). UWF images are captured by Optos with $2600 \times 2048$ pixels of resolution. $1800(81.82 \%)$ and $400(18.18 \%)$ UWF images are set as the training and validation dataset respectively, which are labeled by a qualified ophthalmologist and reviewed by another ophthalmology expert. After the process of resizing and amplifying, the resolution of images is set as $488 \times 488$, and the size of training and validation dataset is 8182 and 1818 respectively. Testing dataset includes 100 UWF images from 50 patients. The in-house data set is properly pruned without sensitive information, like patients' personal data. This study adhered to the Declaration of Shenzhen Aier Eye Hospital, and this research has obtained appropriate permission and approvement from said hospital.

Table 1. Description of the Dataset.

\begin{tabular}{l|c|c|c|c|c}
\hline Dataset Total number & Size of Train dataset & Size of validation dataset & Size of test dataset & Resolution & \\
\hline Original dataset & 2300 & 1800 & 400 & 100 & $2600 \times 2048$ \\
Resized dataset & 2300 & 1800 & 400 & 100 & $448 \times 448$ \\
Amplified dataset & 10000 & 8182 & 1818 & 0 & $448 \times 448$ \\
\hline
\end{tabular}

\section{Data Labeling and preprocessing}

Experts mark the macular fovea on the blank canvas of original UWF images, which has a resolution of $2600 \times 2048$ pixels. A circle is drawn around the marked fovea as the center with a 30 pixels radius. The ophthalmologists are invited from Shenzhen Aier Eye Hospital in China, who are experts with more than 7-year clinical experiences in colorful fundus imaging diagnoses.

As the Figure 2 shows, the preprocessing includes image resizing and amplifying. We resized the original images to $448 \times 448$ pixels and amplified the training dataset for DL models from the size of 2200 to 10000 . The amplifying process includes rotation (set rotation range to 30, width shift (set shift range to 0.3 ), height shift (set shift range to 0.3 ), zoom process (set zoom range to 0.3), horizontal flip and vertical flip. With this process, this study obtained highly accurate labeled training and validation datasets with appropriate sizes.

\section{Methods}

A great number of deep learning models have been implemented for medical useRoy et al. (2017); Li et al. (2020); Girish et al. (2018) since AI research has found its way into medical field. However, as for macular fovea detection tasks, algorithms are required to consider retinal indicator quantifications. Furthermore, when it comes to clinical practice, the invested time is an important factor to be considered to evaluate performanceLi et al. (2020). In this research we compare an FCN architecture model and a 3-layer-based lightweight Unet structure on the task of locating the macular fovea.

\section{Fully Convolutional Network}

Fully Convolutional Network (FCN) is proposed and used by L. Jonathan et al. Long et al. (2015) for semantic segmentation, which means to segment images in a pixel level. In their research they adapted and replace the fully connected layers in some classic CNN models such as AlexNet, with locally connected convolutional layers and form new models of FCN. When talking about Fully Convolutional Network, the Convolutional Neural Network is to be mentioned. As the mainstream technique in image tasks, segmentation methods based on CNN possess the common drawbacks: high computational cost the consequently lower efficiency. Conventional CNN techniques are suitable for almost any kind of image classification or/and regression tasks, they accept whole image input and produce mathematical descriptions (value, possibility). Hence the main difference between CNN and FCN where FCN takes in random (or customized) size of input, and produces correspondent size of output, which presents key 


\section{Data Labeling}

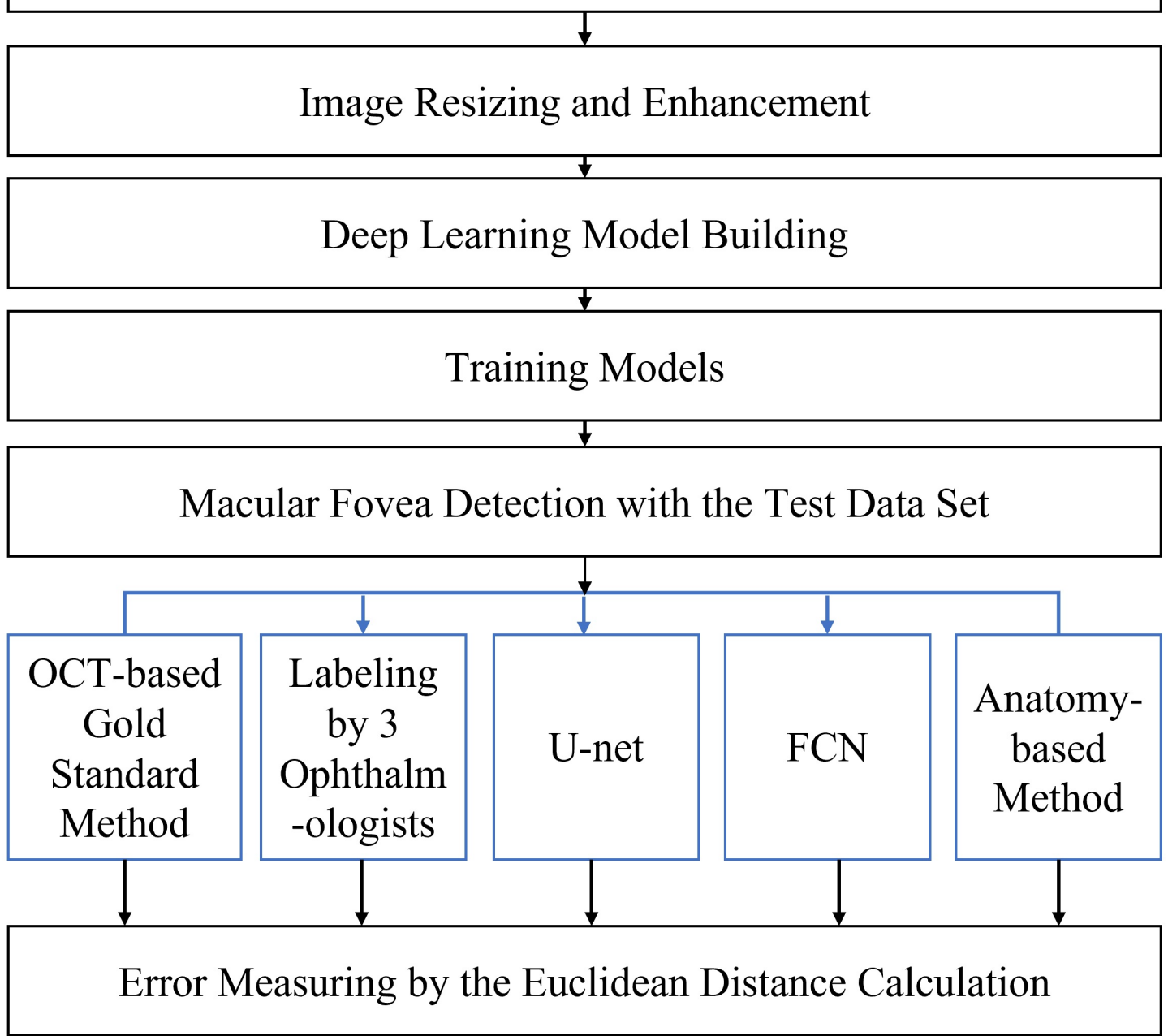

Figure 1. Materials and Methods.

characteristics: end-to-end, pixel-to-pixel learning. Structurally speaking, difference from FCN to CNN is that FCN only uses locally connected layers (pooling, convolution, etc.) instead of fully connected layers at late stage of processing, which requires fewer parameters and would possibly reduce the computational cost comparing to conventional CNN models. As we know, fully connected layers process the image as a whole, while locally connected convolutional layers in FCN process the image as parts, therefore using locally connected convolutional layers allows the convnet to slide through images of larger size (which breaks through the limitation on the size of input image). FCN takes in an arbitrary size image as input, after multiple convolutions and pooling the produced result become smaller and poorer resolution. To restore the processed result back to correspondent sized image, deconvolution is used to up sample for the feature map of the last convolutional layer and produce a prediction for each pixel while also maintain the spatial coordinates from original input image.

Until now some might consider FCN a more efficient solution over some conventional CNN method, but its disadvantages also include that its process excluded the spatial regulation step, which is seen in some common segmentation models, this might lead to possible lack of spatial consistency. And because FCN conducts pixelwise prediction, correlations between pixels could possibly be overlooked.

Unet

Unet is first proposed by Olaf Ronneberger et al. Ronneberger et al. (2015) in 2015, the objective is to achieve segmentation for biomedical images. This model is call U-net because of its symmetric processing 


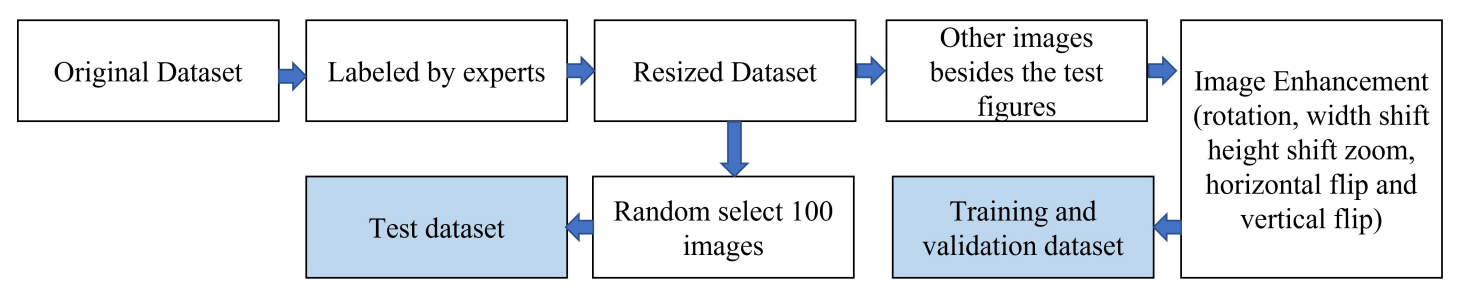

Figure 2. Preprocessing Workflow.

INPUT $=(448,448$ ,3)

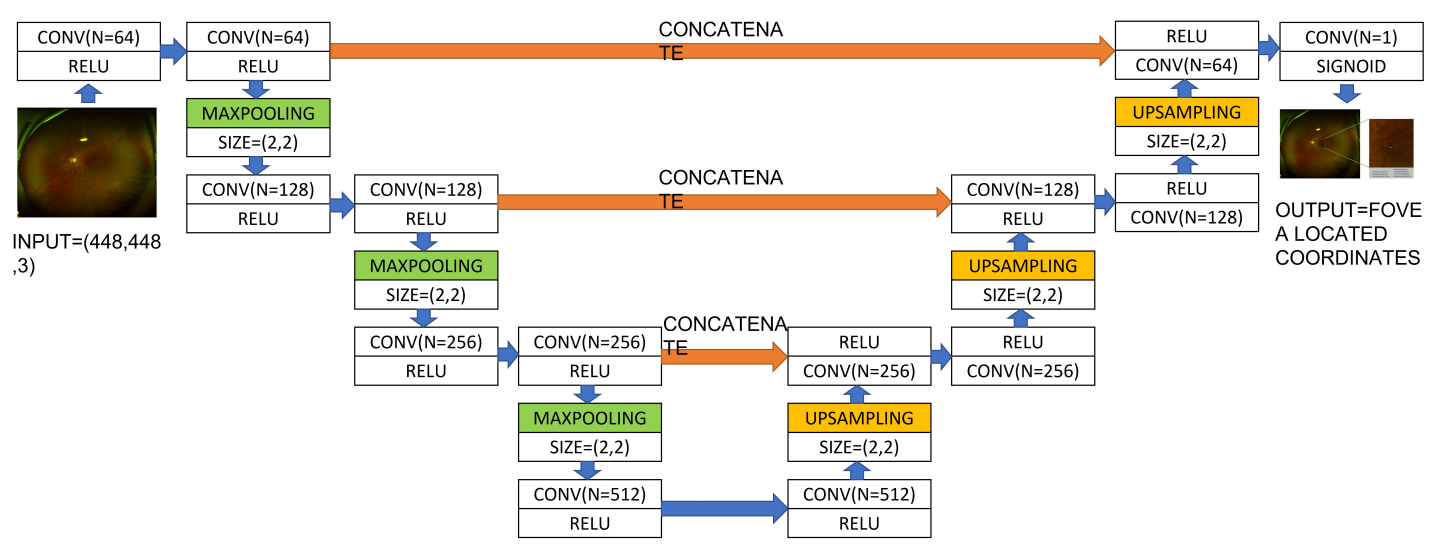

structure resembles the letter ' $U$ '. U-net's mechanism is widely recognized to resemble the mechanism of an encoder-decoder relationship. Two symmetric processes are contained in this architecture, which are encoding of subsampling (feature extraction), and decoding of up sampling and concatenation. In the original research, the size of the input is $224 \times 224$. And during feature extraction, after multiple convolutions and pooling, 4 types of features are produced as $112 \times 112,56 \times 56,28 \times 28$ and $14 \times 14$. Then after deconvolution (up sampling) a new $28 \times 28$ feature map is produced from $14 \times 14$, which will then be combined with the extracted feature map with correspondent size. Progressively after multiple deconvolutions and concatenations, the final produced result will be a same sized image as the input. A difference between the aforementioned FCN and U-net is the step of merging feature maps. FCN achieves it by stacking correspondent pixels of the feature maps, and U-net does it through the concatenation of channels. U-net has been widely implemented on processing medical images, it is also utilized as baseline for many related research.

There are obvious advantages of U-net on specifically medical image processing, considering the nature of medical images. They are usually area-specific and narrow-structured, for example CT and MRI of various cancers, therefore most features extracted from the original image could be important. The way that U-net merge feature maps through correspondent channels could protect the integrity of every valuable feature. On the other hand, medical image datasets are hard to obtain and compile, for some diseases there might not be enough amount of images, and if large structure deep learning models such as DeepLabv3+ are used, overfitting problem could easily occur. Therefore, the simpler structured U-net could possibly better handle the task, also provide the researchers with wider space of modifying and optimizing the model according to their research objectives.

This study proposed a 3-layer-based lightweight Unet structure for fovea detection. The architecture of the proposed network is shown in Figure 3. Comparing to the traditional Unet architecture, the size of the input and output image is defined as $448 \times 448$, through multiple convolutions and max-pooling, 4 types of features are produced as $224 \times 224,112 \times 112,56 \times 56$ and $28 \times 28$. Correspondingly, with convolution and up sampling processes, the output image is resized as $448 \times 448$. The other progress is that, instead of 4-layer of Unet, this study proposed a 3-layer architecture, which is more lightweight and resource-saving.

Figure 3. The architecture of the lightweight U-Net for macular fovea detection. 
memory of 48G, which is with tools of CUDA (v10.0), Python 3.7, tensorflow-gpu (v1.13.2), and keras (v2.2.3).

\section{OCT-induced Method and anatomy-based method}

In the experiment we utilized Ultra-widefield swept-source OCT (UWF-OCT)Takahashi et al. (2019) with depths of $5 \mathrm{~mm}$ and scan widths of $23 \mathrm{~mm}$, which are performed on 50 patients of 100 subjects. This method is utilized by multiple researchers for ophthalmology disease tasks, which is defined as the grounded standard methodTakahashi et al. (2019); Mastropasqua et al. (2019); Takahashi et al. (2021); Schaal et al. (2019).

Another method is based on the anatomy-based location relationship between the optic disk and macular fovea based on UWF. OD is regarded as the brightest area of the fundus images, in the experiment we identify the brightest pixel in UWF, calculate OD mask's width $\left(W_{O D}\right)$ and located the center $\left(X_{O D}, Y_{O D}\right)$. The fovea is located at the left of OD center when it comes to the left eye, otherwise, it is detected on the right. Coordinates $\left(X_{M F}, Y_{M F}\right)$ of macular fovea could be calculated as the following equations 1,2 and 3 . For the left eye, the location of the fovea is located as $\left(X_{M F}^{L}, Y_{M F}\right)$, otherwise, it is detected as $\left(X_{M F}^{R}, Y_{M F}\right)$. The parameters of $\gamma$ and $\beta$ is decided by the experts, who are ophthalmologists and have more than 7 years of experiences in macular fovea detection based on colorful fundus images. The parameters setting is $(\gamma=3.04, \beta=0.409)$.

$$
\begin{aligned}
& X_{M F}^{L}=X_{O D}+\gamma \times W_{O D} \\
& X_{M F}^{R}=X_{O D}-\gamma \times W_{O D} \\
& Y_{M F}=Y_{O D}+\beta \times W_{O D}
\end{aligned}
$$

\section{RESULTS AND DISCUSSION}

100 UWF images are tested by the aforementioned methods of FCN deep learning model, Unet algorithm, anatomy-based method, OCT-induced method and manual marking by three experts. The Euclidean distance between the proposed approach and OCT-induced method results are calculated. OCT-induced method is regarded as the grounded standard in Ophthalmic area. Indicators of mean value, mode value, median value, max value, min value, variation ratio, mean difference, standard deviation, discrete coefficient and the difference between max and min value (range value) are calculated to describe errors.

\section{Results}

As the Table 2 shows, deep learning models show a great advantage on time-consuming performance in the experiment. Marking fovea manually is 200 times as time-consuming as anatomy-based computer-aided method, 2000 times as FCN-based approach, and approximately 20000 times as proposed light-weighted Unet DL method. Thus, computer-aided methods should be preferred to be considered. The proposed method of light-weighted Unet structured DL model is regarded as the most promising method for labor and time costs. Moreover, Euclidean distance error is calculated by measuring fovea detection results

Table 2. Consuming Time Resource of proposed approaches on the Testing Dataset.

\begin{tabular}{l|c|c|c|c|c|c}
\hline Approaches & $\begin{array}{c}\text { Marking by } \\
\text { expert one }\end{array}$ & $\begin{array}{c}\text { Marking by } \\
\text { expert two }\end{array}$ & $\begin{array}{c}\text { Marking by } \\
\text { expert three }\end{array}$ & $\begin{array}{c}\text { Anatomy-based } \\
\text { method }\end{array}$ & $\begin{array}{c}\text { FCN-based } \\
\text { method }\end{array}$ & $\begin{array}{c}\text { Unet-based } \\
\text { method }\end{array}$ \\
\hline $\begin{array}{l}\text { Mean Time-consuming } \\
\text { (seconds) }\end{array}$ & 217.00 & 197.50 & 245.50 & 1.20 & 0.10 & 0.01 \\
\hline
\end{tabular}

of proposed light-weighted Unet-based deep learning approach and other methods with the OCT-UWF grounded method. A descriptive analysis of errors is presented by the 3 , where four findings are extracted. (1) The proposed deep learning method based on Unet algorithm presents great performances with the lowest value of mean value, mode value, max value, min value, mean difference, standard deviation and discrete coefficient, and the second low median value and range value. (2) Comparing measures between ophthalmologist artificial marking (manpower) results, there is a great difference of max and min value of their distance errors, the variation ratio, mean difference standard deviation discrete coefficient and range value are relatively high. This manual marking results show a high inconsistency and discrete 
degree of errors. A pool stability falls on the manual marking method. (3) As for the anatomy-based computer-aided method, the max error is lower than 300, which is also not the highest among the five selected approaches, which indicated that parameters of anatomy-based method are verified to be effective. (4) As far as deep-learning models considered, the proposed Unet-based light-weighted method presents a better performance on $9 / 10$ indicators than the comparable FCN-based method. The max value of the proposed method is 5-time less than the other one. Regarding parameters of other distance-error value descriptive analysis, i.e. the mean value, mode value, median value and min value, and the degree of dispersion (for method stability evaluation), i.e. the mean difference, standard deviation, discrete coefficient and range value, the three-layer Unet algorithm exhibits a better performance with much lower values than FCN-based model.

Table 3. Statistical Measures on Distance Errors of the Testing Dataset.

\begin{tabular}{l|c|c|c|c|c|c|c|c|c|c}
\hline Approaches & $\begin{array}{c}\text { Mean } \\
\text { Value }\end{array}$ & $\begin{array}{c}\text { Mode } \\
\text { Value }\end{array}$ & $\begin{array}{c}\text { median } \\
\text { Value }\end{array}$ & $\begin{array}{c}\text { Max } \\
\text { Value }\end{array}$ & $\begin{array}{c}\text { min } \\
\text { value }\end{array}$ & $\begin{array}{c}\text { Variation } \\
\text { Ratio }\end{array}$ & $\begin{array}{c}\text { Mean } \\
\text { Difference }\end{array}$ & $\begin{array}{c}\text { Standard } \\
\text { Deviation }\end{array}$ & $\begin{array}{c}\text { Discrete } \\
\text { Coefficient }\end{array}$ & $\begin{array}{c}\text { Range } \\
\text { Value }\end{array}$ \\
\hline $\begin{array}{l}\text { Marking by } \\
\text { expert one }\end{array}$ & 25.64 & $\& 18.1$ & 171.68 & 0.98 & 1 & 17.84 & 170.7 & 27.18 & 1.06 & 30.34 \\
\hline $\begin{array}{l}\text { Marking by } \\
\text { expert two }\end{array}$ & 23.91 & 13.97 & 15 & 202.06 & 0.84 & 0.98 & 16.92 & 201.22 & 1.27 \\
\hline $\begin{array}{l}\text { Marking by } \\
\text { expert three }\end{array}$ & 18.72 & 13.26 & 13.72 & 147.51 & 1.72 & 0.98 & 11.3 & 145.79 & 19.36 & 1.03 \\
\hline $\begin{array}{l}\text { Anatomy-based } \\
\text { method }\end{array}$ & 73.74 & $\& 64.94$ & 240.23 & 9.29 & 1 & 36.58 & 230.94 & 47.3 & 0.64 & 1.88 \\
\hline $\begin{array}{l}\text { FCN-based } \\
\text { method }\end{array}$ & 28.83 & 32.02 & 22.36 & 547.17 & 1.41 & 0.97 & 17.81 & 545.76 & 54.27 & 1.88 \\
\hline $\begin{array}{l}\text { Unet-based } \\
\text { method }\end{array}$ & 18.32 & 13.33 & 14 & 100.52 & 0.81 & 0.98 & 10.54 & 99.71 & 15.92 & 0.87 \\
\hline
\end{tabular}

Based on descriptive analysis results of the distance error, a performance matrix analysis is implemented based on radar chart, where the basic analysis rule of indicator calculating of $V_{d}$ is measured by the equation 4. $D_{i}$ is the descriptive distance-error analysis result of comparable macular fovea detection methods in table 3 . Five indicators are considered in this process, which are mean value, median value, max value, min value, mean difference and discrete coefficient. The higher the $V_{d}$ is, the better performance the $D_{i}$ exhibits. Radar chart is also known as the star plot, where the best performance matrix falls on the biggest star. The graphical visualization of radar chart is as the figure 4 shown, stars represent the methods of marking by three ophthalmologists, the anatomy-based computer-aided method, FCN-based DL method and proposed Unet-based light-weighted DL method. Results shows that the proposed method of Unet presents the best performances among the six indicators, where weakest performances fall on Anatomy-based method.

$$
V_{d}=D_{i} /\left(\operatorname{Average}\left(D_{i}\right)\right)
$$

Four UWF images from individual observations are visualized as figure 4. As for UWF figures of right eyes (a and c), the macular fovea is located on the left of the optic disc, on the contrary, the fovea of left eyes (e and g) is located on the right of OD. Macular fovea marking results are displayed in figure b, $\mathrm{d}, \mathrm{f}$ and $\mathrm{h}$ detailly. Three findings are indicated from the figures. (1) Almost all of the fovea detecting results are located near the darkest position on the macular. (2) The error of proposed DL method of light-weighted Unet model is the least, comparing with other method, which is the nearest to the grounded standard of OCT-UWF method. (3) Errors of anatomy-based method results is in the effective range. However, it is still with a high error comparing with other methods.

\section{Discussion}

Macular fovea detection plays a significant role in diagnoses and treatment for fundus disease. With the shortage of ophthalmologists, as well as for patients' considerations, a computer-aided method for fundus lesion detection. OCT-UWF is the most standard way to detect the fovea. However, it costs more time and the resource. Thus, a high efficient, accuracy and stability, and resource-saving (time, equipment, energy, labor, etc.) method is preferred for UWF macular fovea detections. 


\title{
The Radar chart of macular fovea detection methods
}

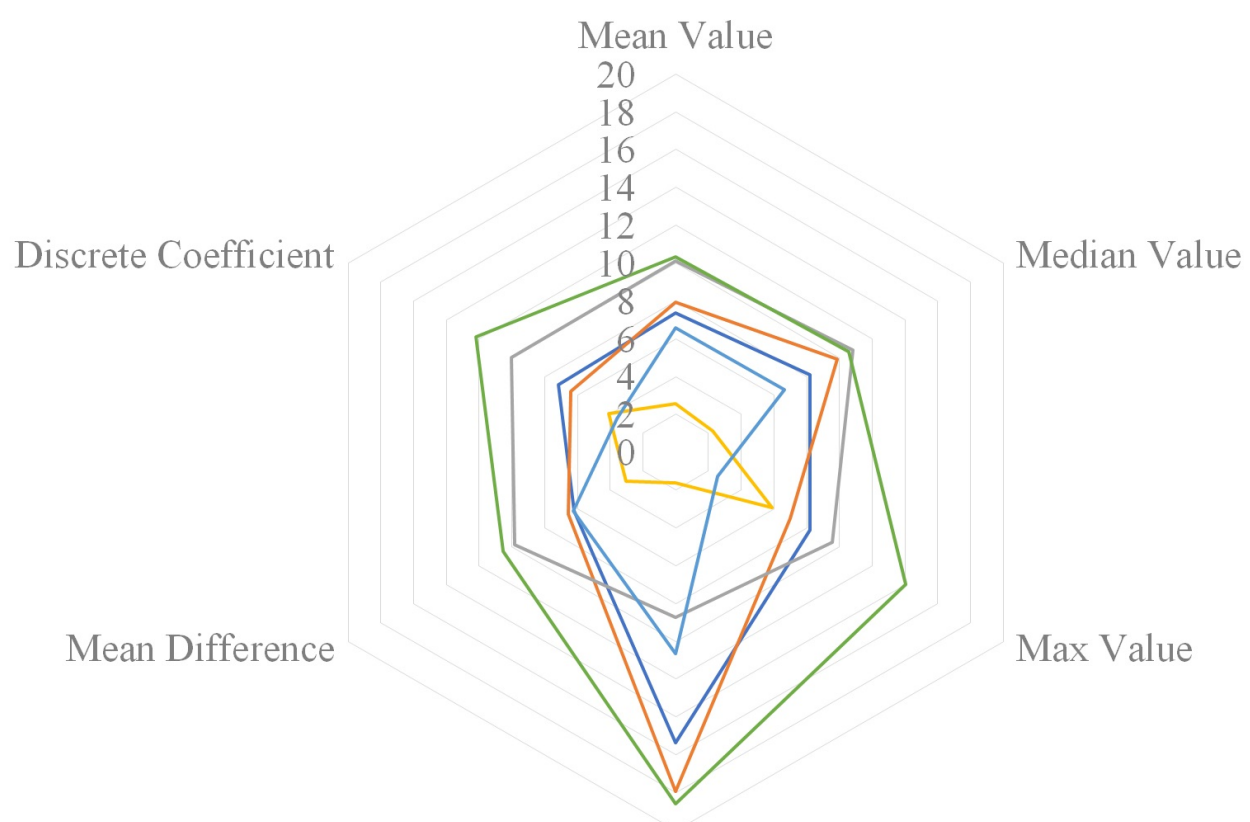

Min value

\begin{abstract}
-Marking by expert one -Marking by expert two -Marking by expert three -Anatomy-based method-FCN-based method -Unet-based method
\end{abstract}

Figure 4. The radar chart result of comparable macular fovea detection methods.

Comparing to the manual marking approach, automatic models exhibit a great strength of efficiency, stability, accuracy and time-saving. The proposed method is a three-layer Unet-structured light-weighted DL algorithm. Results show that not only the proposed method the great advantages of the lowest error and highest stability, but also the most resource-saving approach.

Furthermore, the results verifies that there is an anatomic distance relationship between the OD and the macular fovea. The parameters of anatomy-based method this article set are considered effective. However, considering the high max error and the dispersion degree of this method, there is still some differences between the OD-fovea distance rules for UWF with different eye disease. Moreover, the findings are explored based the 100-sized test dataset, which indicated that a bigger dataset is recommended for the future researches.

\section{CONCLUSIONS}

In this paper, we experimented on a dataset containing 2300 UWF images collected from Shenzhen Aier Eye Hospital in China. With 5 different methods: FCN-based method, Unet-based method, anatomybased method, OCT-induced method and manual marking by three experts, the objective is to find the best performance method for macular fovea detection. OCT-induced method refers to Ultra-widefield swept-source OCT-based method, which is recognized as the grounded standard in Ophthalmic area. Considering the limited resource in the clinical practice, this study proposed a 3-layer-based lightweight Unet architecture for fovea diagnoses. There is a spatial relationship between macula fovea and OD 
center in UWF, a set of parameters to detect the fovea based on the location of OD center is verified to be effective. Comparing to the grounded standard on testing datasets, deep learning models show a great advantage in time-saving performance over other approaches. The manual marking method showed a shortage in performance of resource-saving, accuracy and stability. The proposed lightweight Unet-architecture model performed best on efficiencies, stabilities and precises. Thus, the proposed method of 3-layer lightweight Unet architecture model exhibits a potential to be widely implemented for macular fovea detections in the clinical practice.

The dataset we used contains 2300 UWF images, but it is still insufficient for further optimization and development of new advanced methods. Furthermore the access of UWF images is still extremely limited in current days, for the researchers with less resources and unable to get necessary permits (ethical, legal), further related research will be restricted. With thriving research on deep learning techniques, ophthalmology, and their interdisciplinary studies, inspiration and motivations are needed, so that clinicalused Deep Learning models can be compared and improved continuously. For future research, the development of medical-used DL models and algorithms are expected to be more objective-oriented and also possibly explainable.

\section{PATENTS}

The Chinese patent named "The method of Macular fovea detection" (No.202010199266.9) is related to this project.

\section{SUPPLEMENTARY MATERIALS}

The "data access authorization and medical data ethics supporting document" from Shenzhen Aier Eye Hospital (both of Chinese and English version) of this study is available online at https://github.com/luckanny111/macular

\section{AUTHOR CONTRIBUTIONS}

The conceptualization research and paper writing are contributed by Han Wang, Jie Yang and Yaoyang Wu. The methodology, software, validation, formal analysis and investigation are contributed by Han Wang and Jie Yang. The visualization is responsible by Han Wang and Yutong Duan. The resources and data curation are contributed by Chen Lin, Qingqian Li, Feng Wu and Prof. Lina Huang. This project is supervised by Prof. Lina Huang, Prof. Jiang Liu and Prof. Wencai Du.

\section{ACKNOWLEDGMENTS}

This research was funded by the 2020 Key Technology R \& D Program of GuangDong Province, grant number of ZH01110405180056PWC, Zhuhai Technology and Research Foundation, grant number of ZH01110405180056PWC, Zhuhai Technology and Research Foundation, grant number of ZH22036201210034PWC, Zhuhai Basic and Application Research Project, grant number of ZH22017003200011PWC and The Science and Technology Innovation Committee of Shenzhen City, grant number of JCYJ20200109140820699.

\section{REFERENCES}

Al-Bander, B., Al-Nuaimy, W., Williams, B. M., and Zheng, Y. (2018). Multiscale sequential convolutional neural networks for simultaneous detection of fovea and optic disc. Biomedical Signal Processing Control, 40:91-101.

Bian, X., Huang, Y., Li, X., Niu, X., Wu, J., and Xie, Y. (2020). Automatic optic disc/cup segmentation and glaucoma classification and fovea localization.

Bird, A. (2020). Role of retinal pigment epithelium in age-related macular disease: a systematic review. British Journal of Ophthalmology.

Carmona, E. J., Rincón, M., García-Feijoó, J., and Martínez-de-la Casa, J. M. (2008). Identification of the optic nerve head with genetic algorithms. Artificial Intelligence in Medicine, 43(3):243-259.

Deka, D., Medhi, J. P., and Nirmala, S. (2015). Detection of macula and fovea for disease analysis in color fundus images. In 2015 IEEE 2nd International Conference on Recent Trends in Information Systems (ReTIS), pages 231-236. IEEE. 
Fogel, A. L. and Kvedar, J. (2018). Artificial intelligence powers digital medicine. NPJ digital medicine, $1(1): 1-4$.

Gass, J. and Wilkinson, C. (1972). Follow-up study of presumed ocular histoplasmosis. TransactionsAmerican Academy of Ophthalmology Otolaryngology, 76(3):672.

Girish, G., Thakur, B., Chowdhury, S. R., Kothari, A. R., and Rajan, J. (2018). Segmentation of intraretinal cysts from optical coherence tomography images using a fully convolutional neural network model. IEEE journal of biomedical health informatics, 23(1):296-304.

Goldbaum, M. H., Katz, N., Nelson, M., and Haff, L. (1990). The discrimination of similarly colored objects in computer images of the ocular fundus. Investigative ophthalmology visual science, 31(4):617623.

Kleiner, R. C., Ratner, C. M., Enger, C., and Fine, S. L. (1988). Subfoveal neovascularization in the ocular histoplasmosis syndrome. a natural history study. Retina, 8(4):225-229.

Kranias, G. (1985). Vitreous hemorrhage secondary to presumed ocular histoplasmosis syndrome. Annals of ophthalmology, 17(5):295-8, 302.

Li, M., Wang, Y., Ji, Z., Fan, W., Yuan, S., and Chen, Q. (2020). Fast and robust fovea detection framework for oct images based on foveal avascular zone segmentation. OSA Continuum, 3(3):528-541.

Long, J., Shelhamer, E., and Darrell, T. (2015). Fully convolutional networks for semantic segmentation. In Proceedings of the IEEE conference on computer vision and pattern recognition, pages 3431-3440.

Mastropasqua, R., Viggiano, P., Borrelli, E., Evangelista, F., Libertini, D., Di Antonio, L., and Toto, L. (2019). In vivo mapping of the choriocapillaris in high myopia: a widefield swept source optical coherence tomography angiography. Scientific reports, 9(1):1-6.

Medhi, J. P. and Dandapat, S. (2016). An effective fovea detection and automatic assessment of diabetic maculopathy in color fundus images. Computers in biology and medicine, 74:30-44.

Nagasawa, T., Tabuchi, H., Masumoto, H., Enno, H., Niki, M., Ohara, Z., Yoshizumi, Y., Ohsugi, H., and Mitamura, Y. (2019). Accuracy of ultrawide-field fundus ophthalmoscopy-assisted deep learning for detecting treatment-naïve proliferative diabetic retinopathy. International ophthalmology, 39(10):2153-2159.

Nagiel, A., Lalane, R. A., Sadda, S. R., and Schwartz, S. D. (2016). Ultra-widefield fundus imaging: a review of clinical applications and future trends. Retina, 36(4):660-678.

Niemeijer, M., Abràmoff, M. D., and Van Ginneken, B. (2009). Fast detection of the optic disc and fovea in color fundus photographs. Medical image analysis, 13(6):859-870.

Nussenblatt, R. B. (2010). Ocular histoplasmosis. Uveitis: Fundamentals and Clinical Practice. 4th ed. Edinburgh, UK: Mosby/Elsevier, pages 210-8.

Rivers, M. B., Pulido, J. S., and Folk, J. C. (1992). Ill-defined choroidal neovascularization within ocular histoplasmosis scars. Retina, 12(2):90-95.

Ronneberger, O., Fischer, P., and Brox, T. (2015). U-net: Convolutional networks for biomedical image segmentation. In International Conference on Medical image computing and computer-assisted intervention, pages 234-241. Springer.

Roy, A. G., Conjeti, S., Karri, S. P. K., Sheet, D., Katouzian, A., Wachinger, C., and Navab, N. (2017). Relaynet: retinal layer and fluid segmentation of macular optical coherence tomography using fully convolutional networks. Biomedical optics express, 8(8):3627-3642.

Schaal, K. B., Munk, M. R., Wyssmueller, I., Berger, L. E., Zinkernagel, M. S., and Wolf, S. (2019). Vascular abnormalities in diabetic retinopathy assessed with swept-source optical coherence tomography angiography widefield imaging. Retina, 39(1):79-87.

Schlaegel Jr, T. (1977). Ocular histoplasmosis. Distributed for Grune Stratton, Inc., by Academic Press Inc.(London) Ltd ....

Sedai, S., Tennakoon, R., Roy, P., Cao, K., and Garnavi, R. (2017). Multi-stage segmentation of the fovea in retinal fundus images using fully convolutional neural networks. In 2017 IEEE 14th International Symposium on Biomedical Imaging (ISBI 2017), pages 1083-1086. IEEE.

Sekhar, S., Al-Nuaimy, W., and Nandi, A. K. (2008). Automated localisation of optic disk and fovea in retinal fundus images. In 2008 16th European Signal Processing Conference, pages 1-5. IEEE.

Singh, J., Joshi, G. D., and Sivaswamy, J. (2008). Appearance-based object detection in colour retinal images. In 2008 15th IEEE International Conference on Image Processing, pages 1432-1435. IEEE.

Sinthanayothin, C., Boyce, J. F., Williamson, T. H., Cook, H. L., Mensah, E., Lal, S., and Usher, D. J. D. m. (2002). Automated detection of diabetic retinopathy on digital fundus images. 19(2):105-112. 
Takahashi, H., Tanaka, N., Shinohara, K., Uramoto, K., Yokoi, T., Yoshida, T., and Ohno-Matsui, K. (2021). Importance of paravascular vitreal adhesions for development of myopic macular retinoschisis detected by ultra-widefield oct. Ophthalmology, 128(2):256-265.

Takahashi, H., Tanaka, N., Shinohara, K., Yokoi, T., Yoshida, T., Uramoto, K., and Ohno-Matsui, K. (2019). Ultra-widefield optical coherence tomographic imaging of posterior vitreous in eyes with high myopia. American journal of ophthalmology, 206:102-112.

Walma, D. and Schlaegel, T. (1964). Presumed ocular histoplasmosis. Am J Ophthalmol, 57:107-10.

Yang, J., Fong, S., Wang, H., Hu, Q., Lin, C., Huang, S., Shi, J., Lan, K., Tang, R., and Wu, Y. (2021). Artificial intelligence in ophthalmopathy and ultra-wide field image: a survey. Expert Systems with Applications, page 115068.

Yang, Z., Li, X., He, X., Ding, D., Wang, Y., Dai, F., and Jin, X. (2019). Joint localization of optic disc and fovea in ultra-widefield fundus images. In International Workshop on Machine Learning in Medical Imaging, pages 453-460. Springer. 

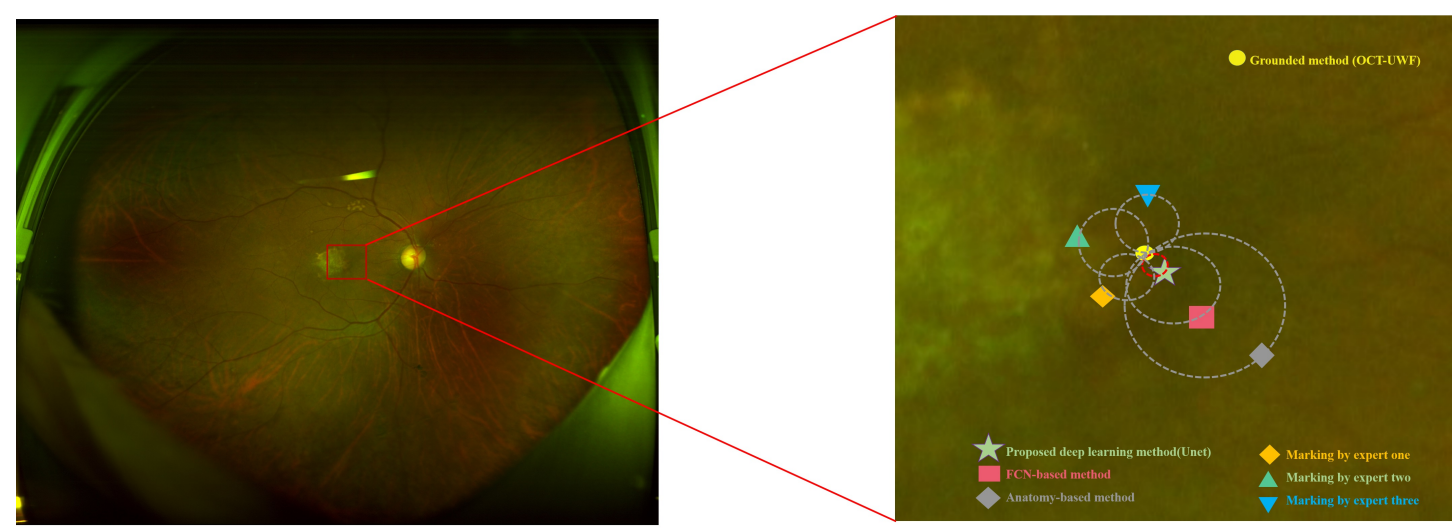

a

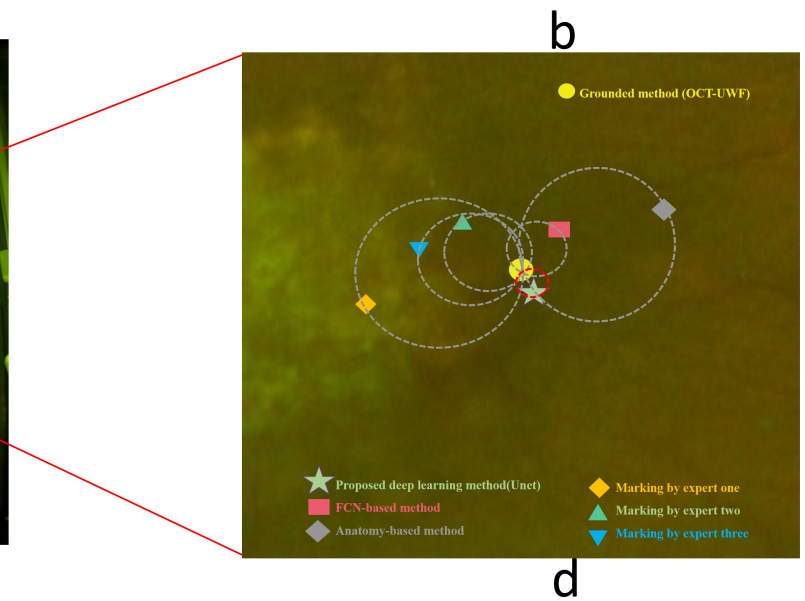

C

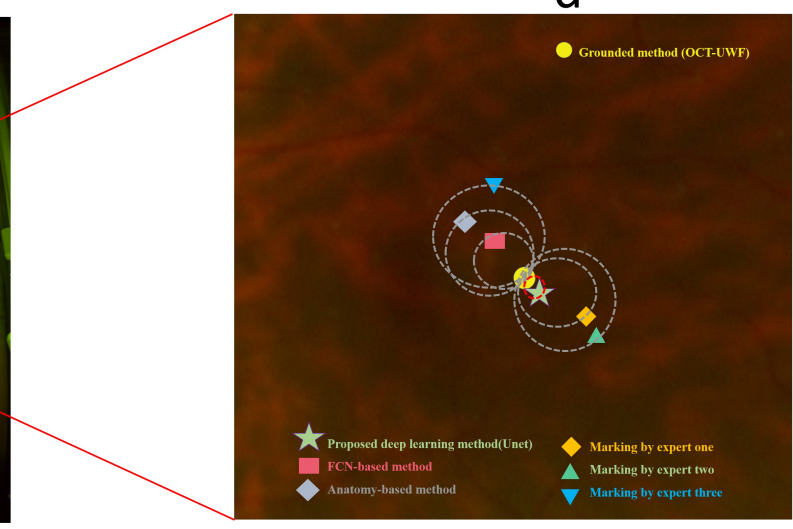

e

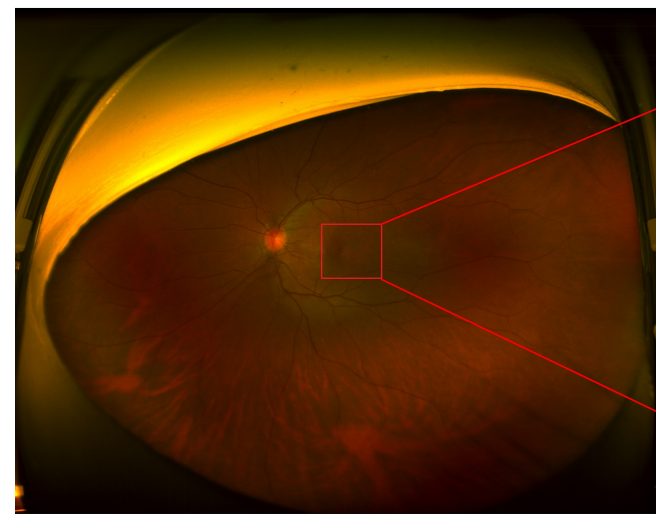

g

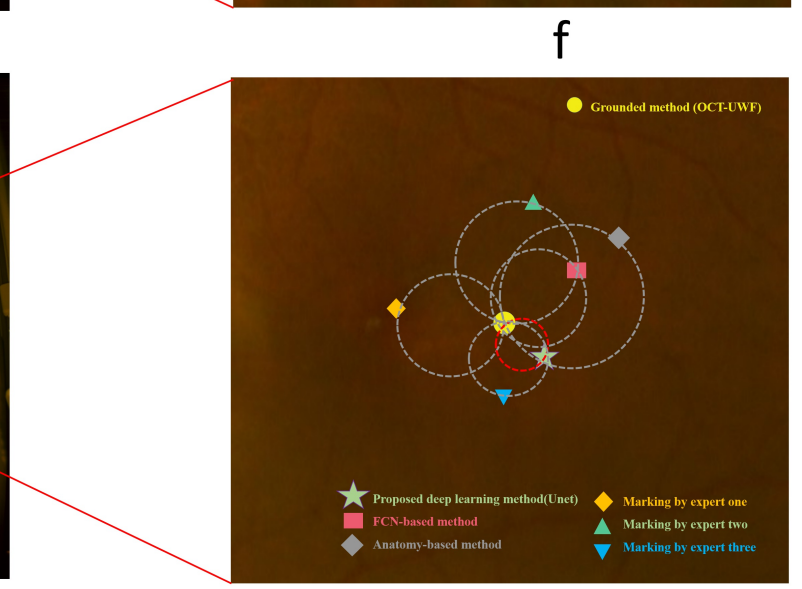

$\mathrm{h}$

Figure 5. The comparison of multiple methods for spatial positioning results of the macular fovea. This figure is related to four UWF from four different observation objects. Figure a and $\mathrm{c}$ is the UWF of $\mathbf{1} \mathbf{3} \mathbf{6} \mathbf{4}$ ? eyes of two individual patients, e and $g$ is the UWF of left eyes of the other two patients. Figure $b, d, e$ and $\mathrm{f}$ are the macular fovea marking results captured from the figure $\mathrm{a}, \mathrm{c}, \mathrm{e}$ and $\mathrm{g}$ respectively. The circle 